\title{
Experimental Demonstration Of Scanned Spin-Precession Microscopy
}

\author{
V. P. Bhallamudi,${ }^{1, *}$ C. S. Wolfe,${ }^{1}$ V. P. Amin,${ }^{2}$ D. E. Labanowski,${ }^{1}$ \\ A. J. Berger ${ }^{1}$ D. Stroud,${ }^{1}$ J. Sinova, ${ }^{2}$ and P. C. Hammel ${ }^{1, \dagger}$ \\ ${ }^{1}$ Department of Physics, The Ohio State University, Columbus, Ohio 43210, USA \\ ${ }^{2}$ Department of Physics and Astronomy, Texas A\&M University, College Station, Texas, USA
}

(Dated: June 30, 2021)

Progress in spintronics has been aided by characterization tools tailored to certain archetypical materials [1-6]. New device structures and materials [7-12] will require characterization tools that are material independent, provide sufficient resolution to image locally-varying spin properties and enable subsurface imaging. Here we report the demonstration of a novel spin-microscopy tool based on the variation of a global spin-precession signal in response to the localized magnetic field of a scanned probe. We map the local spin density in optically pumped GaAs from this spatiallyaveraged signal with a resolution of $5.5 \mu \mathrm{m}$. This methodology is also applicable to other spin properties and its resolution can be improved. It can extend spin microscopy to device structures not accessible by other techniques, such as buried interfaces and non-optically active materials, due to the universal nature of magnetic interactions between the spins and the probe.

Precession in a magnetic field is a hallmark characteristic of a magnetic moment, which makes it a powerful discriminant of spin-related phenomena in spintronic studies; this is exploited in Hanle effect measurements [13]. It can also result in loss of spin information in the presence of unwanted magnetic fields, especially spatially varying ones. However, in this report we show that the spin precession due to the spatially inhomogeneous magnetic field of a micromagnetic probe $(\mu P)$ can be employed to encode local spin information into a spatially integrated measurement. This information can then be decoded using standard deconvolution techniques.

To demonstrate our technique we first measure a signal proportional to the globally-averaged spin density, $\Sigma$, as a function of the $\mu P$ 's position. From this measurement we can then obtain a quantity proportional to the unperturbed spin density (i.e. the steady state density in the absence of the probe), $\boldsymbol{\rho}$, by deconvolving the signal from a theoretical or experimentally-determined Precessional Response Function (PRF). The PRF captures the response of the spins to the magnetic environment they experience. We repeat this process in the presence of an applied uniform transverse magnetic field to further demonstrate our understanding of this microscopy tool.

We generate $\boldsymbol{\rho}=\rho\left(\mathbf{r}_{s}\right) \hat{\mathbf{z}}$, in a GaAs membrane via optical pumping [13], where $\mathbf{r}_{s}=\left(x_{s}, y_{s}, 0\right)$ is the position within the sample which we treat as two dimen- sional. Due to precession the steady state spin density in the presence of any magnetic field will be different from $\boldsymbol{\rho}$, and we will denote it by $\mathbf{S}$. We measure a globally averaged spin-PhotoLuminescence (PL) signal [13], $\Sigma \propto \int_{-\infty}^{\infty} S_{z} \mathrm{~d}^{2} \mathbf{r}_{s}$, where $S_{z}$ refers to the $\hat{\mathbf{z}}$ component of S.

This signal is measured while the pump laser is scanned relative to the $\mu P$ in the $\hat{\mathbf{x}}$ and $\hat{\mathbf{y}}$ directions. For our uniform sample, this is equivalent to scanning the $\mu P$ relative to a fixed pump, and henceforth we will regard this to be the case. The field $\mathbf{B}_{p}$ of the $\mu P$ modifies the precession behavior of the spins (see Fig. 1). This field depends on $\mu P$ 's position. A spatially uniform transverse magnetic field, $B_{t} \hat{\mathbf{x}}$, may also be applied to further tailor this precession. More details of the experimental set-up and measurement techniques are presented in the Supplementary Information (SI).

Fig. 2b shows the measured PL intensity (which is proportional to $\rho$ ) for a particular $\rho=\rho_{c}$, which will be used later for obtaining the PRF. Panels $\mathbf{c}$ and $\mathbf{d}$ show the corresponding $\Sigma_{c}$, for $B_{t}=0 \mathrm{~T}$ and $0.145 \mathrm{~T}$ respectively, as a function of the $\mu P$ 's position, $\mathbf{r}_{p}=\left(x_{p}, y_{p}, z_{p}\right)$.

An expression for $\Sigma$ in the limit of small diffusion (which our data shows is a reasonable approximation for this experiment; more details of the derivation in the $\mathrm{SI})$ is given by:

$$
\begin{aligned}
\Sigma\left(\mathbf{r}_{p}, B_{t}\right) & \propto \int_{\infty}^{\infty} S_{z}\left(\mathbf{r}_{s}, \mathbf{r}_{p}\right) \mathrm{d}^{2} \mathbf{r}_{s} \\
& \propto \int_{\infty}^{\infty} H_{B}\left(\mathbf{R}, B_{t}\right) \rho\left(\mathbf{r}_{s}\right) \mathrm{d}^{2} \mathbf{r}_{s} \\
& =H_{B}\left(\mathbf{R}, B_{t}\right) * \rho\left(\mathbf{r}_{s}\right)
\end{aligned}
$$

where, * represents a convolution, $\mathbf{R}=\mathbf{r}_{p}-\mathbf{r}_{s}$ and

$$
H_{B}=\frac{1}{1+\theta_{B}^{2}} \quad \text { and } \quad \theta_{B}^{2}\left(\mathbf{R}, B_{t}\right)=\frac{\left(\gamma \tau_{s} B_{\perp}\right)^{2}}{1+\left(\gamma \tau_{s} B_{\|}\right)^{2}} .
$$

$H_{B}$ is the PRF and is the signal which would be collected for a spatial delta function injection. $B_{\perp}$ and $B_{\|}$ are respectively the magnitudes of the perpendicular and parallel components of the total field, $\mathbf{B}=\mathbf{B}_{p}(\mathbf{R})+B_{t} \hat{\mathbf{x}}$, experienced by the spins. The components are defined with respect to $\hat{\mathbf{z}}$, the orientation of injected spins. The gyromagnetic ratio is denoted by $\gamma$ and $\tau_{s}$ is the spin relaxation time.

As seen from the previous equations, $S_{z}$ is decreased by $B_{\perp}$ because it causes the spins to precess away from 


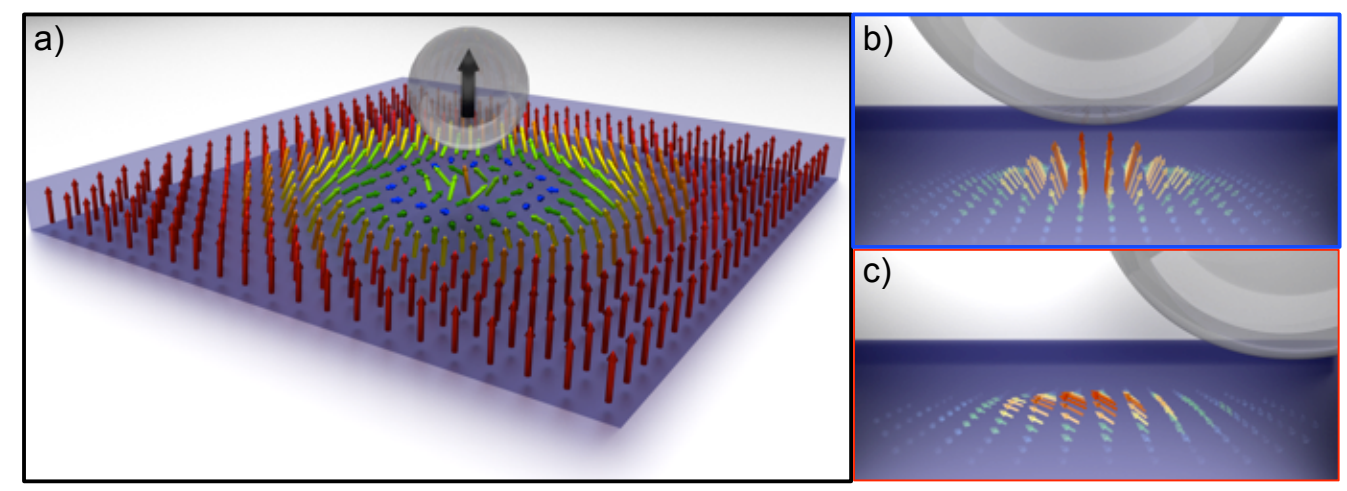

FIG. 1. The simulations shown illustrate the key physics underlying Spin Precession Imaging; the inhomogeneous field of a micromagnetic probe $\mu P$ (sphere with black arrow, indicating the magnetization direction) generates a well-understood spatially-varying dephasing of spins. Spins that are injected into a semiconducting sample reach a steady-state density resulting from a combination of the local spin properties, the injected density, and the magnetic field due to the $\mu P$. The arrows in all figures represent the steady-state spin density vectors $\mathbf{S}$. The color scale represents the $\hat{\mathbf{z}}$ component (parallel to the orientation of the injected spin) of the spins. a) Demonstrates the case of a spatially-uniform injection density and highlights the full spatial-extent of the $\mu P$ 's influence. Strong transverse fields from the probe dephase spins in an annular disk centered beneath the probe. Parallel fields immediately below the probe protect spins from dephasing. b) and c) show simulations identical to panel a) except for the case of a spatially confined injection profile, corresponding to two different $\mu P$ positions. Panels $\mathbf{b}$ and c correspond to the blue and red dots in Fig. 2 c respectively.

the injected direction, resulting in a dephasing of the ensemble. On the other hand $B_{\|}$keeps the spins from tipping away from the injection direction, resulting in a small $\theta_{B}$ and large $S_{z}$. We can view $\theta_{B}$ as an effective dephasing factor $[14,15]$.

$B_{\|}$and $B_{\perp}$ have distinct spatial variation in our experiments, and the consequences of their competing effects are evident in Fig. 3, where we show line scans (along $\hat{\mathbf{x}}$ and $\hat{\mathbf{y}}$ ) for several values of $B_{t}$. Also shown are fits obtained from Eqn. 1 in which the probe is modeled as a point dipole with a moment $\mathbf{m}=m_{p} \hat{\mathbf{z}}$ located a height $z_{p}$ above the sample (see Fig. 3 caption and the SI for more details).

The peak, marked by vertical green dashed lines at $x$ or $y=0$, occurs when the $\mu P$ is located directly above the point of maximum injected spin density. At this point there is a maximum in $B_{\|}(\sim 0.8 \mathrm{~T})$ from the $\mu P$ that preserves $S_{z}$. When the pump is far from the $\mu P$, the signal decreases with increasing $B_{t}$ with a Lorentzian line shape (as expected in a Hanle measurement) whose halfwidth, $B_{1 / 2}=\left(\gamma \tau_{s}\right)^{-1}$, is $0.0111 \mathrm{~T}$ for our experiments.

A second peak (blue dashed line) seen in the line scans along $\hat{\mathbf{x}}$ occurs where the $\hat{\mathbf{x}}$ component of the field from the $\mu P$ cancels $B_{t}$. As $B_{t}$ is increased, this point occurs closer to the $\mu P$ where its field is stronger.

The fits indicate the effectiveness of Eqn. 1 in describing our data. The sensitivity of the global signal to spins at different locations relative to the $\mu P$, described by the convolution, forms the basis for imaging.

To obtain an unknown spin density, $\rho_{u}$, from our measured signal, the PRF needs to be known. The PRF can be obtained theoretically or from experimental data, if we have a known $\rho$. For the latter, we use the camera data shown in Fig $2 \mathbf{d}$ as being proportional to $\rho_{c}$. Then $H_{B}\left(\mathbf{R}, B_{t}\right)=\Sigma_{c}\left(\mathbf{r}_{p}, B_{t}\right) \circledast \rho_{c}\left(\mathbf{r}_{s}\right)$, where we use $\circledast$ to indicate a deconvolution process. We use the Wiener algorithm [16] to implement the deconvolution. The resulting experimental PRFs for both low and high $B_{t}$ are shown in Fig 4. For comparison the theoretical PRFs are also shown; more details for obtaining them are presented in the SI.

To test the fidelity of our imaging process, we now use the experimental PRF for an unknown $\rho_{u}$. The measured $\Sigma_{u}$, at low and high fields, is presented in panels $\mathbf{a}$ and $\mathbf{c}$ of Fig. 5. We then extract $\rho_{u}\left(\mathbf{r}_{s}\right)=$ $\Sigma_{u}\left(\mathbf{r}_{p}, B_{t}\right) \circledast H_{B}\left(\mathbf{R}, B_{t}\right)$. The extracted spin densities are shown in panels $\mathbf{b}$ and $\mathbf{d}$ of the same figure. Also shown (panel e), for independent verification of our imaging technique, is a camera image for the PL $\left(\propto \rho_{u}\right)$. The linecuts present a more quantitative comparison of the extracted and measured data.

The ability to extract the spin density with both high and low $B_{t}$ shows the exclusion of spurious effects, such as reflectivity changes as a function of the $\mu P$ 's position, in our data. Also, $B_{t}$ provides a knob to optimize the PRF to suit particular imaging needs. High field imaging might provide a more intuitive PRF for the case of global detection. Low-field imaging maybe more useful for non-local electrical devices, where a large transverse field would dephase spins before they reach the detector.

The line scans shown in Fig. 3 provide us a measure of the spatial resolution, $\zeta$, in our experiment. A Gaussian fitting of the narrowest lobe gives us $\zeta \leq 5.5 \mu \mathrm{m}$. It is an upper bound that is being set by the feature size we are imaging. As in magnetic resonance imaging, the magnetic field gradient, $\kappa$, sets the ultimate resolution in 


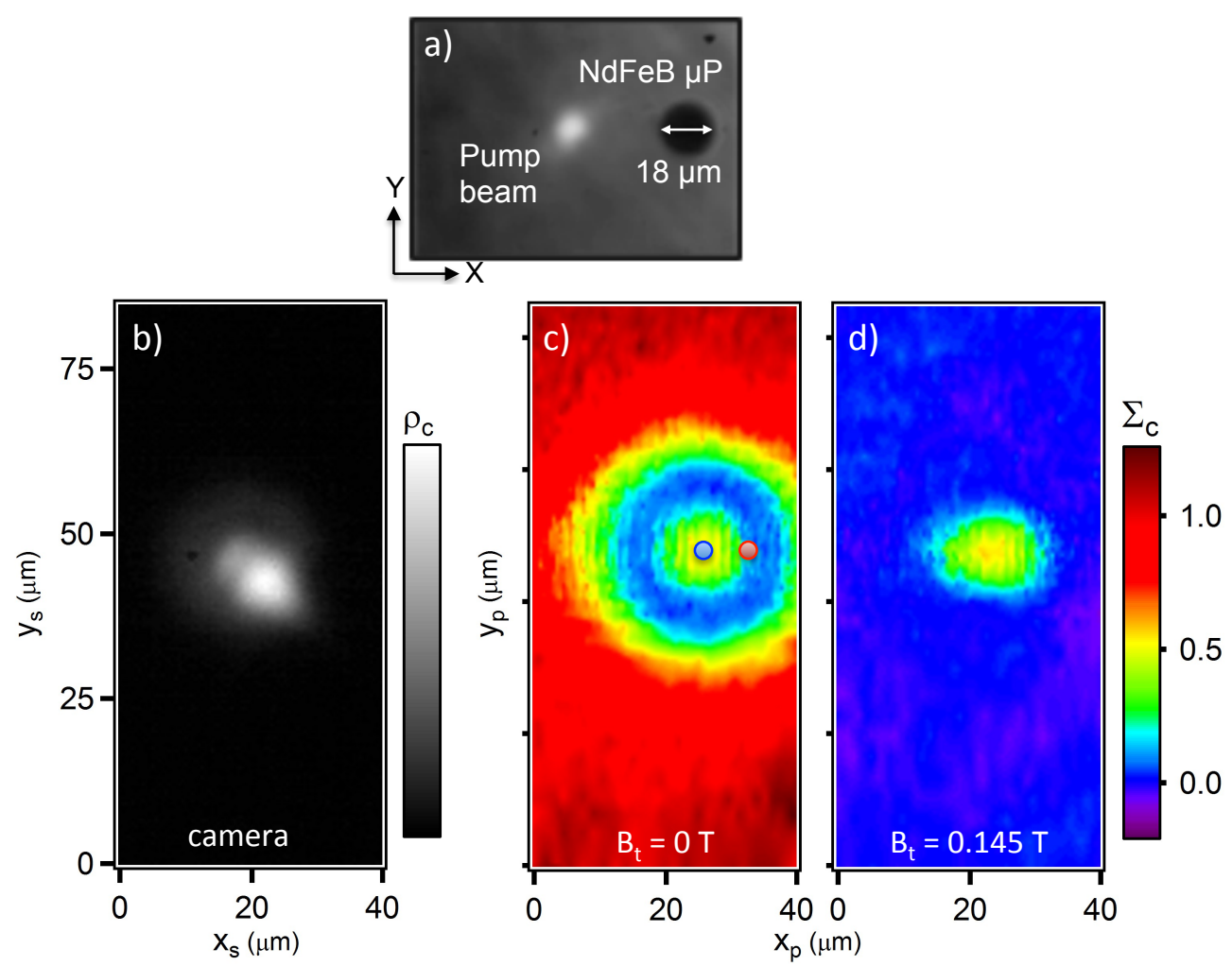

FIG. 2. Spatial maps of the injection beam profile from the optical pump, the injected spin profile and the response of the global spin signal to probe position relative to the pump. a) Spin-insensitive camera image showing the PL spot created by the pump beam in a $1 \mu \mathrm{m}$ thick GaAs membrane. Also visible is the the NdFeB micromagnetic probe $\mu P$, magnetized along the sample normal and glued to the membrane on the side opposite to that of the pump beam. An additional uniform magnetic field $B_{t} \hat{\mathbf{x}}$ may be applied. In the experiment the pump beam is scanned relative to the $\mu P$ using an objective mounted on translation stages. b) Camera image of PL that corresponds to (and is proportional to) injection profile $\rho_{c}$. c) The measured spin signal $\Sigma_{c}$ (colorbar) corresponding to the injection profile from panel $\mathbf{b}$, at $B_{t}=0$. The location of each pixel corresponds to the relative position between the $\mu P$ and the pump beam; a blue and red dot are included to provide two example cases. The location of the blue dot corresponds to a $\mu P$ position directly above the injection beam; this configuration results in a spin density mostly pointing along the $\hat{\mathbf{z}}$ direction (Fig. 1b) and gives a large signal. The red dot corresponds to a $\mu P$ position 8 $\mu \mathrm{m}$ away from the center of the beam; spins precess away from the $\hat{\mathbf{z}}$ direction due to large perpendicular fields (Fig. 1c) and thereby reduce the signal. d) $\Sigma_{c}$ for a large $B_{t}=0.145 \mathrm{~T}$.

the absence of diffusion, $\zeta=B_{1 / 2} / \kappa[14]$. Gradients of up to $\sim 4 \times 10^{6} \mathrm{~T} / \mathrm{m}$ have been reported recently [17], which are at least an order of magnitude larger than in this experiment, and should enable much finer resolution.

Resolution will be limited by diffusion and the unavoidable reduction of the spin signal as the detected volume shrinks. While diffusion can degrade the resolution, the spatial precessional response can be numerically analyzed to obtain valid and useful spatially resolved data. For large enough gradients, sub-diffusion length and subdiffraction limit resolution should be achievable. Images are obtained in the presence of spin diffusion by MRI [18$20]$; this should be feasible for spin precession imaging as well.

In summary, we have demonstrated a new technique for imaging spin properties using the precessional response of spins to a micromagnetic probe's field. While we have imaged the variations in the spin density, the technique is more general since the response of the spins is sensitive to a variety of spin characteristics including spin lifetime and gyromagnetic ratio. Work is underway to generalize the technique presented here using scannable probes mounted on cantilevers. Due to the magnetic nature of interaction between the probe and the spins, which can extend through layers of a heterostructure and a few microns deep, this tool should enable subsurface imaging. This technique should be applicable to a wide variety of materials because it relies on proven spin polarization detection techniques. With optical detection it can enhance imaging resolution, and with electrical detection it can enable imaging where none exists at present.

Funding for this research was provided by the Center for Emergent Materials at the Ohio State University, an NSF MRSEC (Award Number DMR-0820414). We wish to thank Cristian Cernov for creating the rendered images presented in this article and the SI. 


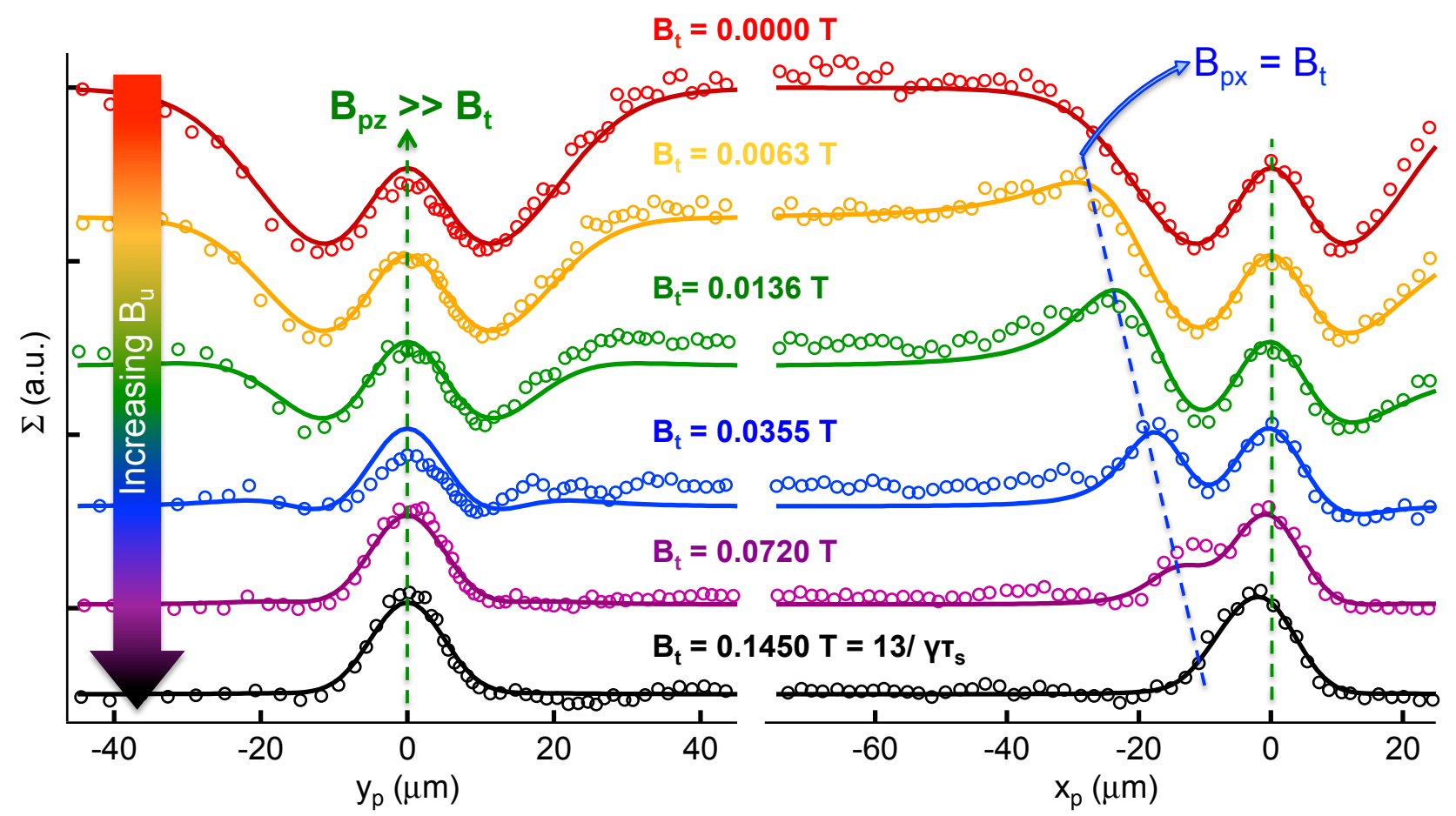

FIG. 3. Spin signal $\left(\Sigma_{c}\right.$, corresponding to $\left.\rho_{c}\right)$ for various $B_{t}$ plotted as a function of the relative position between the pump and the $\mu P$ along the $\hat{\mathbf{y}}$ (left) and the $\hat{\mathbf{x}}$ (right) directions. The data at various $B_{t}$ are offset vertically for clarity of presentation. The open circles are experimental data while the solid lines represent fits obtained from Eqn. 1 using $m_{z}=2 \times 10^{-9} \mathrm{~J} / \mathrm{T}$, $z_{p}=8 \mu \mathrm{m}$, and $\tau_{s}=2.33 \mathrm{~ns}$. The spin lifetime $\tau_{s}$ is obtained from Hanle measurements (see SI) and the $\mu P$ radius $\left(z_{p}\right)$ is taken to be $9 \mu \mathrm{m}$. The peaks marked by the green dashed lines result when the peak in $\rho_{c}$ lies directly under the $\mu P$. The net parallel field directly under the $\mu P$ is mostly due to $B_{p z}$ (the $\hat{z}$ component of the $\mu P$ 's field) and exceeds the net transverse field (mostly given by $B_{t}$ ); this results in a large $\Sigma($ Eqn. 1). The second set of peaks seen in scans along the $\hat{\mathbf{x}}$ direction (blue dashed line) occur when $B_{p x}=-B_{t}$ ( $B_{p x}$ is the $\hat{\mathbf{x}}$ component of $\mu P$ 's field).
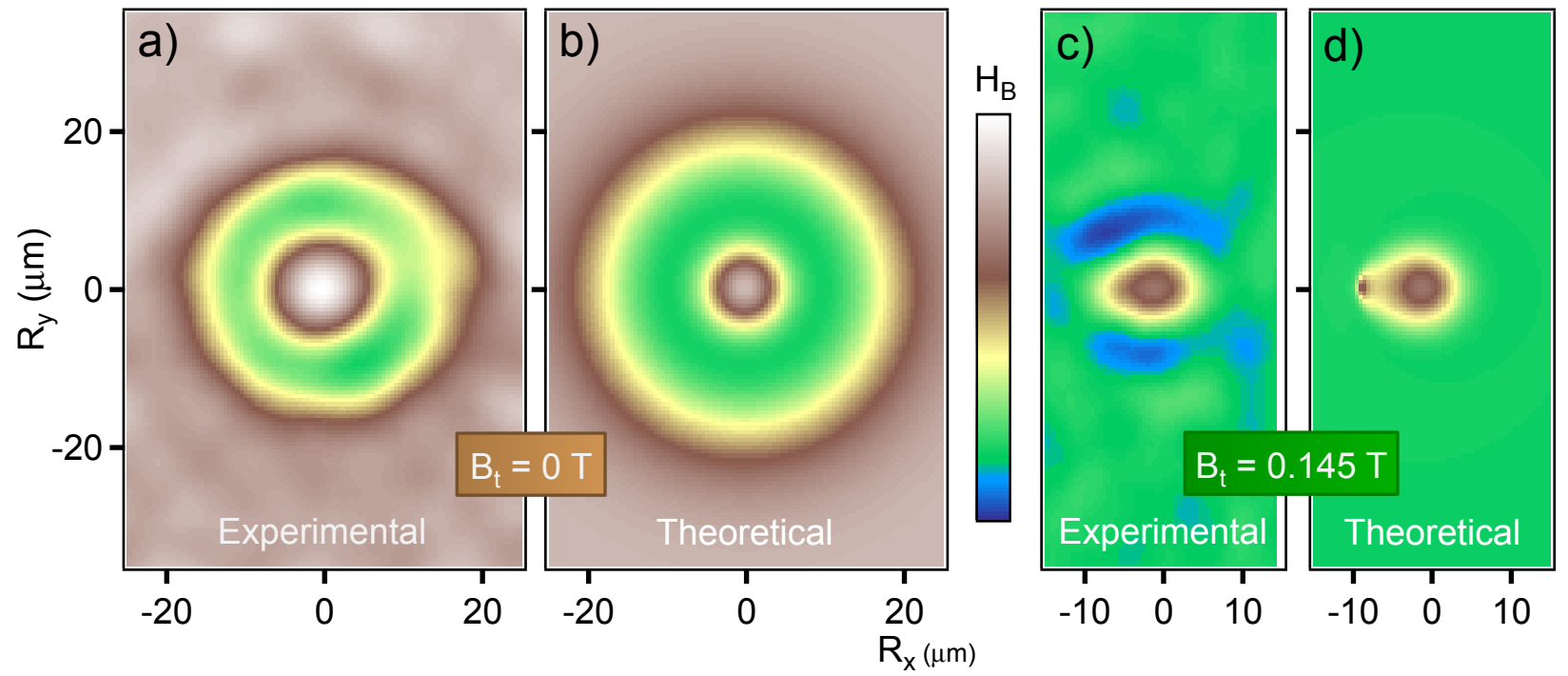

FIG. 4. a) Precessional Response Function obtained through Wiener deconvolution of $\rho_{c}$ (Fig. 2b) from $\Sigma_{c}\left(B_{t}=0\right)($ Fig. $2 \mathbf{c})$. b) Theoretically derived PRF obtained from Eqn. 1, using the dipole moment from the fits in Fig. 3 . c) and d) similar experimental and theoretical PRFs for $B_{t}=0.145 T$. The experimental PRF data has been normalized and offset to highlight the match between theory and experiment. 


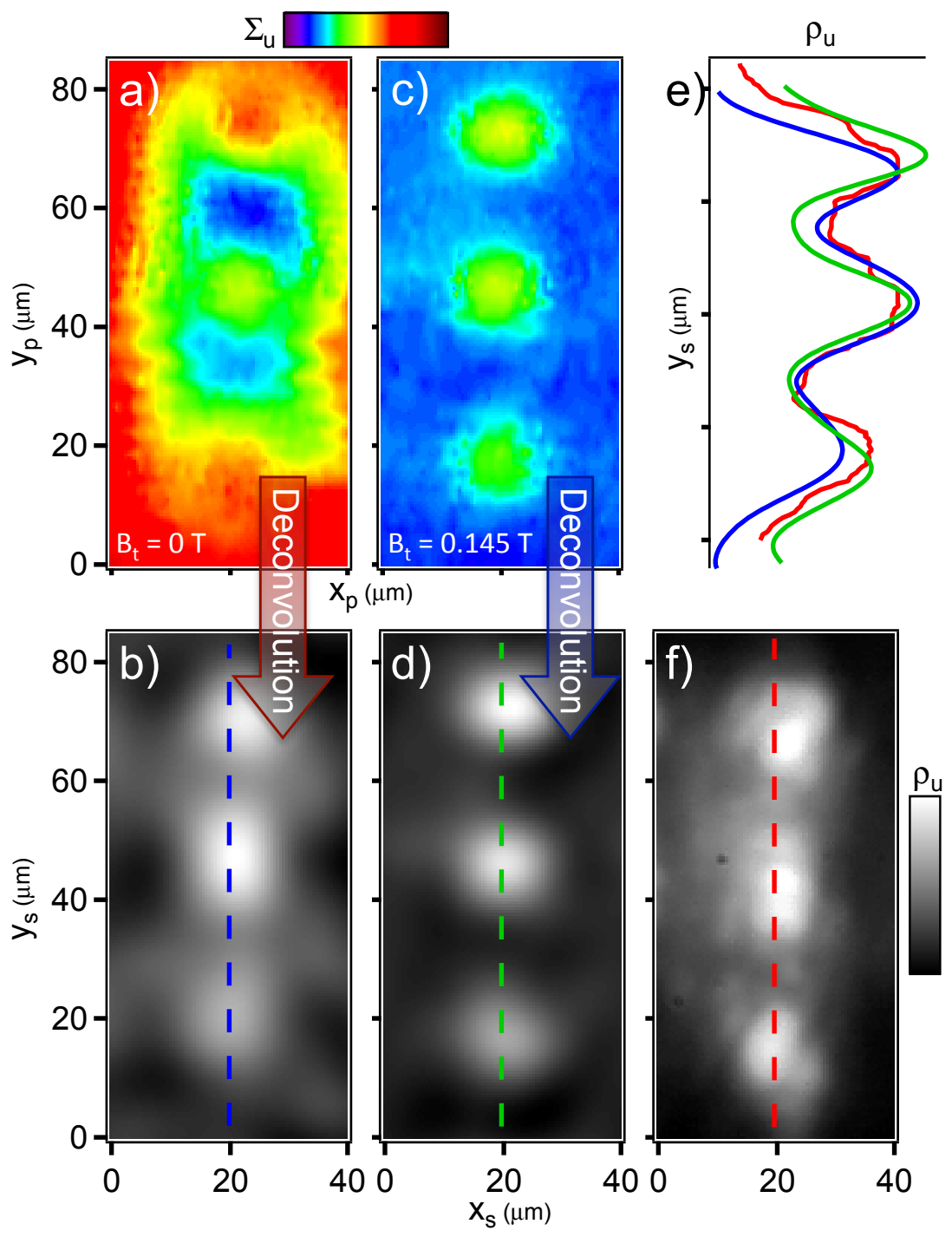

FIG. 5. Obtaining the spatial variation of an unknown spin density from the measured signal $\Sigma$ : a) Spin signal $\Sigma_{u}$ measured for a spin density profile, $\rho_{u}$ (more information on how it was produced given in the SI), with $B_{t}=0 \mathrm{~T}$. b) An image of $\rho_{u}$ extracted from the Wiener deconvolution of $\Sigma_{u}$ (panel a) using the experimental $H_{B}\left(B_{t}=0\right)$ (Fig. 4a) as the deconvolution kernel. c) and d) Similarly measured $\Sigma_{u}$ and extracted $\rho_{u}$ respectively for $B_{t}=0.145 \mathrm{~T}$. e) Line cuts of $\rho_{u}$ taken along the dashed lines in panels $\mathbf{b}$ (blue) and $\mathbf{d}$ (green). Also shown in red is a line cut from an independent camera image of $\rho_{u}$ shown in panel $\mathbf{f}$.

* bhallamudi.1@osu.edu

† hammel@physics.osu.edu

[1] F. Pulizzi, ed., Nat Mater (Insight Issue:Spintronics), vol. 11 (Nature Publishing Group, 2012), URL http: //dx.doi.org/10.1038/nmat3327.

[2] I. Žutić, J. Fabian, and S. D. Sarma, Rev. Mod. Phys. (2004), URL http://link.aps.org/doi/10. 1103/RevModPhys.76.323.

[3] D. D. Awschalom and M. E. Flatte, Nature Phys. 3, 153 (2007), ISSN 1745-2473, URL http://dx.doi.org/10. $1038 /$ nphys 551 .

[4] F. Jedema, H. Heersche, A. Filip, J. Baselmans, and B. van Wees, Nature 416, 713 (2002), URL http://www. nature.com/nature/journal/v410/n6826/ full/410345a0.htm.

[5] S. Crooker, M. Furis, X. Lou, C. Adelmann, D. Smith, C. Palmstrom, and P. Crowell, Science 309, 2191 (2005), URL http://www.sciencemag.org/cgi/ content/abstract/309/5744/2191.

[6] X. Lou, C. Adelmann, S. A. Crooker, E. S. Garlid, J. Zhang, K. S. M. Reddy, S. D. Flexner, C. J. Palmstrom, and P. A. Crowell, Nature Phys. 3, 197 (2007), URL http://www.nature.com/nphys/journal/ v3/n3/full/nphys543.html.

[7] T. Sasaki, T. Oikawa, T. Suzuki, M. Shiraishi, Y. Suzuki, and K. Noguchi, Appl. Phys. Lett. 96, 122101 
(2010), URL http://link.aip.org/link/APPLAB/v96/ i $12 / \mathrm{p} 122101 / \mathrm{s} 1 / \mathrm{html}$.

[8] K. Pi, W. Han, K. M. McCreary, A. G. Swartz, Y. Li, and R. K. Kawakami, Phys. Rev. Lett. 104, 187201 (2010), URL http://prl.aps.org/abstract/PRL/v104/ i18/e187201.

[9] I. Appelbaum, B. Huang, and D. J. Monsma, Nature 447, 295 (2007), ISSN 0028-0836, URL http://dx.doi. org/10.1038/nature05803.

[10] H. C. Koo, J. H. Kwon, J. Eom, J. Chang, S. H. Han, and M. Johnson, Science 325, 1515 (2009), URL http://www. sciencemag.org/cgi/content/abstract/ 325/5947/1515.

[11] S. P. Dash, S. Sharma, R. S. Patel, M. P. de Jong, and R. Jansen, Nature 462, 491 (2009), supplementary information, URL http://www.nature.com/ nature/journal/v462/n7272/full/nature08570.html.

[12] K. Olejnik, J. Wunderlich, A. C. Irvine, R. P. Campion, V. P. Amin, J. Sinova, and T. Jungwirth, Phys. Rev. Lett. 109, 076601 (2012), URL http://link.aps.org/ doi/10.1103/PhysRevLett.109.076601.

[13] F. Meier and B. P. Zakharchenya, Optical Orientation (North-Holland, Amsterdam, 1984), ISBN 9780444867414.

[14] V. P. Bhallamudi, A. J. Berger, D. E. Labanowski,
D. Stroud, and P. C. Hammel, Journal of Applied Physics 111, 013902 (pages 4) (2012), URL http://link.aip. org/link/?JAP/111/013902/1.

[15] R. Jansen, B. C. Min, S. P. Dash, S. Sharma, G. Kioseoglou, A. T. Hanbicki, O. M. J. van 't Erve, P. E. Thompson, and B. T. Jonker, Phys. Rev. B 82, 241305 (2010).

[16] J. Russ, The Image Processing Handbook (Taylor \& Francis, 2011), ISBN 9781439840450, URL http://books. google.com/books?id=gxXXRJWfEsoC.

[17] C. L. Degen, M. Poggio, H. J. Mamin, C. T. Rettner, and D. Rugar, Proc. Nat. Acad. Sci. USA 106, 1313 (2009), ISSN 0027-8424.

[18] J.-D. Tournier, F. Calamante, D. G. Gadian, and A. Connelly, NeuroImage 23, 1176 (2004), ISSN 10538119, URL http://www.sciencedirect.com/science/ article/pii/S1053811904004100.

[19] P. Basser, J. Mattiello, and D. LeBihan, Biophysical Journal 66, $259 \quad$ (1994), ISSN 00063495, URL http://www.sciencedirect.com/science/ article/pii/S0006349594807751.

[20] K. M. Jansons and D. C. Alexander, Inverse Problems 19, 1031 (2003), URL http://stacks.iop.org/ $0266-5611 / 19 / i=5 / a=303$. 


\title{
Supplementary Information: Experimental Demonstration Of Scanned Spin-Precession Microscopy
}

\author{
V. P. Bhallamudi, ${ }^{1, *}$ C. S. Wolfe, ${ }^{1}$ V. P. Amin, ${ }^{2}$ D. E. Labanowski, ${ }^{1}$ \\ A. J. Berger, ${ }^{1}$ D. Stroud, ${ }^{1}$ J. Sinova, ${ }^{2}$ and P. C. Hammel ${ }^{1, \dagger}$ \\ ${ }^{1}$ Department of Physics, The Ohio State University, Columbus, Ohio 43210, USA \\ ${ }^{2}$ Department of Physics and Astronomy, Texas A\&M University, College Station, Texas, USA
}

(Dated: June 30, 2021) 


\section{SAMPLE}

The sample is a $1 \mu \mathrm{m}$ thick (and $\sim 3 \mathrm{~mm} \times \sim 3 \mathrm{~mm}$ ) n-GaAs (001) membrane prepared by etching away the substrate underneath an MOCVD grown epitaxial layer. Fig. S1 shows the growth structure, where the top layer forms the membrane. A nominally lattice-matched InGaP layer is grown under this device layer to act as a stop-etch layer during the fabrication process. SIMS analysis (EAG labs) shows that the doping level of Si in the device layer is $\sim 1.4 \times 10^{22} \mathrm{~m}^{-3}$. This is below the metal-insulator transition level for GaAs. Thus the samples are insulating during our low temperature $(<20 \mathrm{~K})$ measurements. This supports the lack of significant diffusion in our measurements.

To prepare for the etching, the device layer side was glued to a $0.5 \mathrm{~mm}$ thick single crystal (0001)-Sapphire substrate $(5 \mathrm{~mm} \times 5 \mathrm{~mm})$, whose c-axis orientation was chosen to reduce birefringence during the polarization dependent measurements. The sample was glued using Epotek 301-2 optical epoxy to reduce PL background.

The substrate was etched away following a recipe given elsewhere ${ }^{1}$. The etch stops at the InGaP layer because of the high etch selectivity. The InGaP is then etched away using $\mathrm{HCl}$. After the etching process, a NdFeB micromagnetic particle was glued to the membrane (on the side opposite to the sapphire and where the substrate used to be) using micro-manipulators, under an optical microscope.

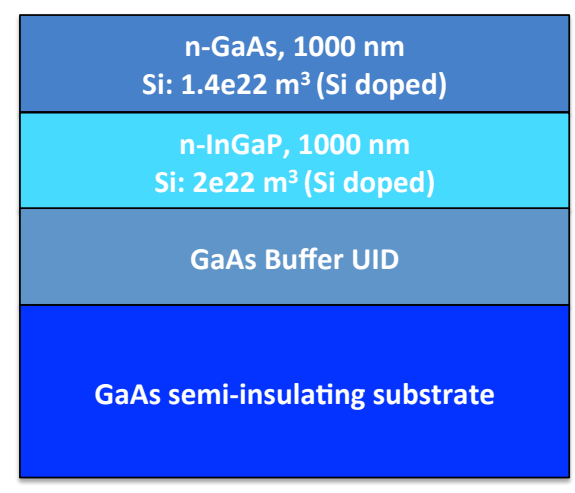

FIG. S1. The MOCVD growth structure for the GaAs sample.

\section{THE OPTICAL SET-UP}

Spins are injected into the GaAs membrane using optical spin injection, which is a well established technique. The detection is based on measuring the circular polarization of the PL, which in turn is proportional to the spin polarization within the sample. The set-up used for optical pumping and the spin-PL measurements is shown in Fig. S2. Also, provided in Table S1 is a detailed list of the various optical components used and their purpose.

The Electro-Optic Modulator (EOM) allows us to modulate the polarization of light, and thus modulate the spin polarization within the sample, between $+\hat{\mathbf{z}}$ and $-\hat{\mathbf{z}}$, and enable lock-in measurements. The EOM modulates the $780 \mathrm{~nm}$ pump between two linear polarization states. These are converted into the two circular states by the the Quarter Wave Plate (QWP). The circularly polarized laser light is focused on the sample (which sits inside an optical cryostat at a temperature of $17 \mathrm{~K}$ ) using an objective. A wire mesh is placed in the path of the pump beam to produce the spin density profile $\rho_{u}$ (see main text). When producing different spin density profiles, the pump power was adjusted to keep the Hanle half-width far away from the $\mu P$ equal.

The circularly polarized light injects spins due to a combination of spin-orbit coupling and optical selection rules. The resulting PL due to the recombination of spin-polarized carriers is circularly polarzied. This PL (at the band edge of GaAs, $819 \mathrm{~nm}$ ) is then collimated by the objective and converted back into the linear states by the QWP. The Wollaston prism splits the two orthogonal linear states; each of which is then collected by a separate photodiode that are part of a diode-bridge circuit. More details of how the photodiode signal is processed is presented in the next section.

The end of a multi-mode fiber is placed $\sim 1 \mathrm{~mm}$ away from the sample (on the side opposite to laser injection, i.e. on the $\mu P$ side). The fiber is used for illuminating the sample with unpolarized broadband light (from a halogen lamp) to assist with camera imaging and tracking of the $\mu P$ during the measurements. The back illumination gives negligible spin signal for our lock-in measurements. 


\begin{tabular}{|c|c|c|}
\hline S.no & Description & Comments \\
\hline A & GaAs sample & see section I for more details \\
\hline $\mathrm{B}$ & Electromagnet & Power supply controlled via DAQ card \\
\hline $\mathrm{C}$ & Optical crystat & Operated at $17 \mathrm{~K}$ \\
\hline $\mathrm{D}$ & Objective lens & plan fluor $10 \mathrm{X}$ \\
\hline $\mathrm{E}$ & Quarter-wave plate & \\
\hline $\mathrm{F}$ & Dichroic mirror & Reflects pump towards the sample, but attentuates it during transmission \\
\hline G & Notch Filter & For $780 \mathrm{~nm}$ \\
\hline $\mathrm{H}$ & Cube beam splitter & Non-polarizing \\
\hline I & Focusing lens & $\mathrm{f}=100 \mathrm{~mm}$ \\
\hline $\mathrm{J}$ & Wollaston prism & Separates the two linear polarization components \\
\hline $\mathrm{K}$ & Bandpass filter & Centered at $820 \mathrm{~nm}, 10 \mathrm{~nm}$ width \\
\hline $\mathrm{L}$ & Photodiode bridge & The difference channel is amplified by a factor of 100000 \\
\hline $\mathrm{M}$ & focusing lens & $\mathrm{f}=200 \mathrm{~mm}$ \\
\hline $\mathrm{N}$ & Cube beam splitter & \\
\hline $\mathrm{O}$ & Long pass filter & cut off at $850 \mathrm{~nm}$ \\
\hline $\mathrm{P}$ & CMOS camera & \\
\hline $\mathrm{Q}$ & Photodiode & To measure spin-insensitive DC level of the PL \\
\hline $\mathrm{R}$ & Wire mesh & \\
\hline $\mathrm{S}$ & Electro-optic modulator & Modulated polarization of light \\
\hline $\mathrm{T}$ & Polarizer & transmits vertical polarization, as needed for the EOM operation \\
\hline $\mathrm{U}$ & Polarizer & Allows for fine control of pump power \\
\hline $\mathrm{V}$ & ND filters & \\
\hline $\mathrm{W}$ & Pump laser & $780 \mathrm{~nm}$, stable laser source, intensity modulated \\
\hline $\mathrm{X}$ & Function generator & used to provide modulation signal for EOM, square, $f_{\text {mod }} \sim 73 \mathrm{~Hz}$ \\
\hline $\mathrm{Y}$ & Voltage preamplifier & Amplifies the A-B channel of the dide bridge, Gain of 200 \\
\hline $\mathrm{Z}$ & Voltage preamplifier & Amplifies the B channel of the dide bridge, Gain of -1000 \\
\hline $\mathrm{AA}$ & Voltage preamplifier & Amplifies the A channel of the diode bridge, Gain of 1000 \\
\hline $\mathrm{AB}$ & Lock-in amplifier & Demodulates the pump power modulation, typical time const. $200-500 \mathrm{~ms}$ \\
\hline $\mathrm{AC}$ & Lock-in amplifier & Demodulates the EOM modulation, typical time const. $200-500 \mathrm{~ms}$ \\
\hline $\mathrm{AD}$ & Function generator & used to provide modulation signal for pump intensity, square, $f_{\text {mod }} \sim 1100 \mathrm{~Hz}$ \\
\hline $\mathrm{AE}$ & Computer & Controls the instruments and performs data acq., thru GPIB and DAQ card \\
\hline
\end{tabular}

TABLE S1. A list of the various optical components and electronic instruments used in the experiment. The s.no correspond to the letters in Fig. S2 and S3.

Also, a variety of optical filters are used in various parts of the optical set-up to remove unwanted light from reaching the photodiodes or the camera. Please see Table S1 for more details.

\section{SCANNING AND DATA COLLECTION}

The data shown in Fig. 2b,c and Fig. 4a,c of the main text are obtained by scanning the objective (which in turn scans the pump beam relative to the sample) using motorized translation stages. The position of the $\mu P$ relative to the beam was tracked using a home built software solution. The Labview-based software uses the camera image and pattern recognition algorithms from National Instruments. This measured position in pixels of the camera image was converted into microns using a SEM image of the $\mu P$ as a calibration.

Fig. S3 shows the instrumentation and hardware signal processing used for obtaining the spin signal from the photodiode voltages. The signals from the photo-diode bride allow us to compute the average steady state spin polarization within the sample, $\Sigma \propto \frac{V_{R}-V_{L}}{V_{R}+V_{L}}$, where the subscripts refer to right or left circular polarization of the PL. The difference signal, $V_{R}-V_{L}$, is the lockin signal due to the modulation of the polarization using the EOM. $V_{R}+V_{L}$ is measured as a different lock-in signal by modulating the power of the pump laser. The normalization by the sum 


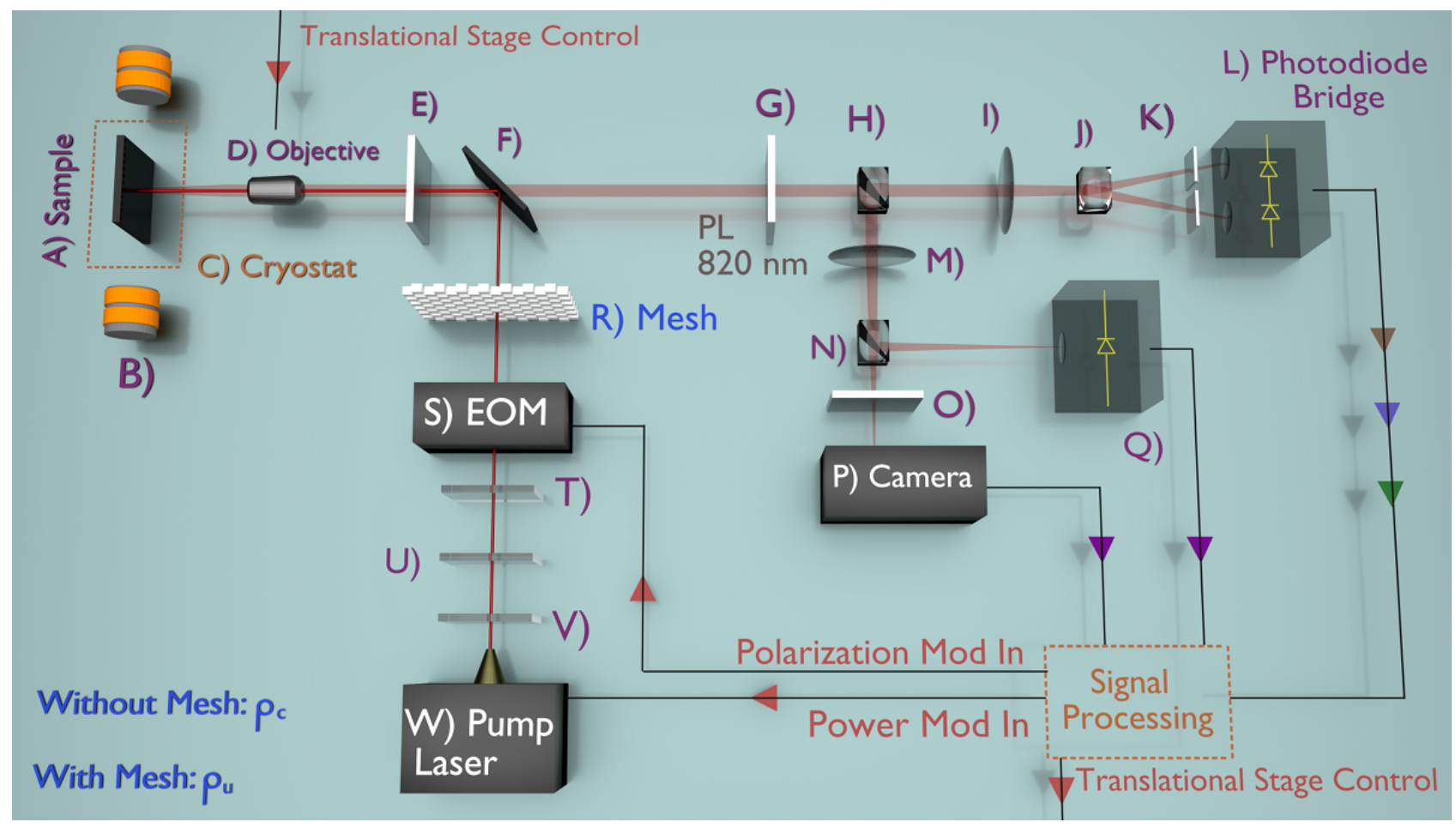

FIG. S2. The optical set-up for the optical pumping and spin PL detection. Please see Table S1 and sec. II for more details of the various components.

of the two circular components removes spurious reflectivity changes in the signal, as seen in Fig. S4.

The step size of the translation stages is somewhat variable. To allow for further data analysis, we have interpolated the $2 \mathrm{D}$ scan data (but not the line scan data) using Igor Pro software to have the signal on a uniform spatial grid. The data shown in the main text is the interpolated data. Further, all the data has been normalized such that $\Sigma$ far away from $\mu P$ at zero field and maximum field are taken to be 1 and 0 respectively. Also, for the line scans the position values were offset to bring the central peak to $x$ or $y=0$.

\section{SPIN DYNAMICS IN A NON-MAGNETIC MATERIAL}

In a two-dimensional non-magnetic semiconductor, the spin density may be governed by the following equation of motion $^{2}$

$$
\frac{\partial \mathbf{S}}{\partial t}=\mathbf{G}+D \nabla^{2} \mathbf{S}+\zeta(\mathbf{E} \cdot \nabla) \mathbf{S}+\gamma \mathbf{B} \times \mathbf{S}-\frac{\mathbf{S}}{\tau_{s}}
$$

where all vector fields $(\mathbf{S}, \mathbf{G}, \mathbf{E}, \mathbf{B})$ are, in general, functions of the spatial coordinate $\mathbf{r}_{s}=\left(x_{s}, y_{s}, 0\right)$ within the sample and time $t$. The spin density is given by $\mathbf{S}$; each component of this vector field gives the difference between spin "up" and "down" particle densities in that direction. The first term on the R.H.S. of Eqn. S1, G, represents the rate at which spin density is externally injected and related to the intensity of the pump light. The second and third terms dictate the diffusion and drift of the spins respectively, where $D$ is the diffusion constant, $\mathbf{E}$ is the electric field, and $\zeta$ the electron (or hole) mobility. We assume no difference in diffusivity between opposite-spin carriers in any direction. The fourth term gives the precession of spin density around a net magnetic field $\mathbf{B}$, with $\gamma=g \mu_{B} / \hbar$ being the gyromagnetic ratio; where $g$ is the g-factor, $\mu_{B}$ is the Bohr magneton, and $\hbar$ the reduced Planck constant. The final term represents spin relaxation with a spin lifetime of $\tau_{s}$.

We study the case in which $\mathbf{E}=\mathbf{0}, \tau_{s}$ is spatially uniform, and $\mathbf{B}$ and $\mathbf{G}$ vary spatially but not in time. We also consider an injection rate constrained to the $z$-axis, i.e. $\mathbf{G}=G_{z} \hat{z}$. From a device point-of-view, one usually measures the steady-state solution of spin density $\mathbf{S}(x, y, t \rightarrow \infty)$; in what follows we will only consider this solution.

In the limit where diffusion is negligible, Eqn. S1 possesses a purely algebraic solution, which can be written more 


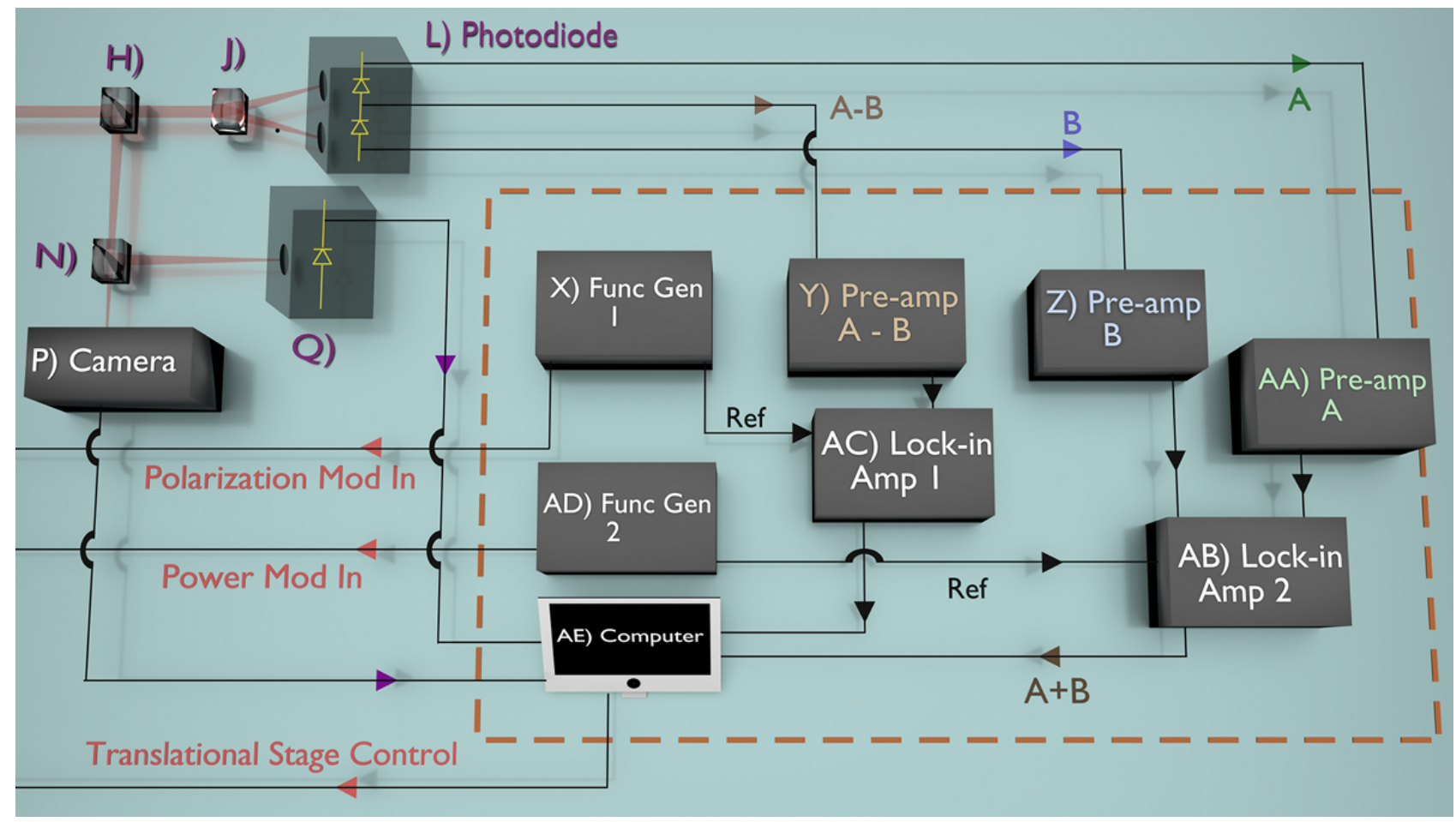

FIG. S3. Schematic of the signal processing done to obtain the measured signal. Please see Table S1 and sec. III for more details of the various components.

conveniently by introducing the following notation

$$
\mathcal{B}=\left(\begin{array}{ccc}
0 & B_{z}^{*} & -B_{y}^{*} \\
-B_{z}^{*} & 0 & B_{x}^{*} \\
B_{y}^{*} & -B_{x}^{*} & 0
\end{array}\right)
$$

where we have applied a scaling to $\mathbf{B}$ (and its vector components $B_{x}, B_{y}, B_{z}$ ), $\mathbf{B}^{*}=\gamma \tau_{s} \mathbf{B}$.

The steady state solution, $\partial \mathbf{S} / \partial t=0$, for the case of $D=0$ is then given by

$$
\mathbf{S}(\mathbf{r})=\left[I-\mathcal{B}\left(\mathbf{r}_{s}\right)\right]^{-1} \rho\left(\mathbf{r}_{s}\right)
$$

where, $\rho\left(\mathbf{r}_{s}\right)=G_{z}\left(\mathbf{r}_{s}\right) \tau_{s}$. It should be noted that $\boldsymbol{\rho}$ is the steady state spin density in the absence of any magnetic field and is the quantity that we are interested in measuring in this experiment from a signal that is averaged in $\mathbf{r}$.

Seen in the above equation is that fact that the absence of both drift and diffusion eliminates all coupling between neighboring positions (which in general are coupled due to derivative operators). Thus, in this particular limit, the steady-state solution is determined algebraically by the magnetic field values at a given position, and does not need to be solved using the full differential equation. The solutions, based on Eqn. S2, for the various vector components of $\mathbf{S}\left(\mathbf{r}_{\mathbf{s}}\right)$ are determined by $B$ and $\rho$ only at that $\mathbf{r}_{s}$; they are given by,

$$
\begin{aligned}
S_{x} & =\frac{B_{x}^{*} B_{z}^{*}-B_{y}^{*}}{1+B_{x}^{* 2}+B_{y}^{*^{2}}+B_{z}^{* 2}} \rho \\
S_{y} & =\frac{B_{y}^{*} B_{z}^{*}+B_{x}^{*}}{1+B_{x}^{* 2}+B_{y}^{*^{2}}+B_{z}^{* 2}} \rho \\
S_{z} & =\frac{1+B_{z}^{* 2}}{1+B_{x}^{* 2}+B_{y}^{* 2}+B_{z}^{* 2}} \rho,
\end{aligned}
$$




\section{THE MEASURED SIGNAL}

In our experiment the measured signal may be described by

$$
\Sigma \propto \int_{A} S_{z}(\mathbf{r}) d x_{s} d y_{s}
$$

where $A$ represents the area of detection and can be assumed to extend to infinity. The magnetic field, $\mathbf{B}$, in our experiment is provided by the field from a micromagnetic probe $(\mu P), \mathbf{B}_{p}$, and a spatially uniform field $B_{t} \hat{\mathbf{x}}$. Assuming the $\mu P$ to be a point dipole, the field experienced by the spins, at position $\mathbf{r}_{s}$ within the sample, is given by

$$
\begin{aligned}
\mathbf{B}(\mathbf{R}) & =\mathbf{B}_{p}(\mathbf{R})+B_{t} \hat{x} \\
& =\frac{\mu_{0}}{4 \pi} \frac{3 \mathbf{R}(\mathbf{m} \cdot \mathbf{R})-\mathbf{m} R^{2}}{R^{5}}+B_{t} \hat{\mathbf{x}} .
\end{aligned}
$$

Here, $\mathbf{R}=\mathbf{r}_{s}-\mathbf{r}_{p}$ is the relative spatial vector between the position of the $\mu P, \mathbf{r}_{p}$, and the position of the spins within the sample; $\mathbf{m}$ is the moment of the $\mu P$ and $\mu_{0}$ is the permeability of free space.

Substituting Eqns. S3 and S5 into Eqn. S4, we can write the measured signal as

$$
\begin{aligned}
\Sigma\left(\mathbf{r}_{p}\right) & \propto \int_{\infty}^{\infty} \int_{\infty}^{\infty} S_{z}\left(\mathbf{r}_{s}, \mathbf{r}_{p}\right) d x_{s} d y_{s} \\
& \propto \int_{\infty}^{\infty} \int_{\infty}^{\infty}\left(\frac{1+B_{z}^{* 2}(\mathbf{R})}{1+B_{x}^{*}(\mathbf{R})^{2}+B_{y}^{*}(\mathbf{R})^{2}+B_{z}^{*}(\mathbf{R})^{2}}\right) \rho\left(\mathbf{r}_{s}\right) d x_{s} d y_{s} \\
& \propto H_{B}(\mathbf{R}) * \rho\left(\mathbf{r}_{s}\right) .
\end{aligned}
$$

The $\star$ operator denotes a two-dimensional convolution integral and $H_{B}$ is the Precessional Response Function $(P R F)$, which may be written in the following manner to emphasize the physics at play

$$
\begin{aligned}
H_{B}(\mathbf{R}) & =\frac{1+B_{z}^{* 2}(\mathbf{R})}{1+B_{x}^{*}(\mathbf{R})^{2}+B_{y}^{*}(\mathbf{R})^{2}+B_{z}^{*}(\mathbf{R})^{2}} \\
& =\frac{1}{1+\theta_{B}^{2}(\mathbf{R})}
\end{aligned}
$$

where the effective dephasing factor $\theta_{B}$ is given by

$$
\theta_{B}^{2}(\mathbf{R})=\frac{\gamma^{2} B_{\perp}(\mathbf{R})^{2} \tau_{s}^{2}}{1+\gamma^{2} B_{\|}(\mathbf{R})^{2} \tau_{s}^{2}}
$$

where $B_{\|}=\sqrt{B_{x}^{2}+B_{y}^{2}}$ and $B_{\perp}=B_{z}$ refer to the parallel and perpendicular components of the total field $\mathbf{B}$, with respect to the injected spin direction $\hat{\mathbf{z}}$.

\section{FITTING THE LINE SCANS}

The fits to the line scans shown in Fig. 3 of the main text were done using the convolution equation shown in Eqn. 1 of the main text (same as Eqn. S6). The various parameters for the fit were either measured independently or constrained by measured quantities. The $B_{1 / 2}=1 / \gamma \tau_{s}$ was obtained using Hanle measurement done far away from the $\mu P$. This Hanle data is shown in Fig. S5, along with the Lorenztian fitting done to obtain the halfwidth. The fitted $B_{1 / 2}=111 \mathrm{G}$ corresponds to a lifetime of $2.33 \mathrm{~ns}$ assuming a g-factor of -0.44 for GaAs. No diffusion $(D=0)$ was assumed. A moment of $2 \times 10^{-9} \mathrm{~J} / \mathrm{T}$ and a moment height of $8 \mu \mathrm{m}$ were used. These parameters provide a better fit (in terms of mean squared error) than $3 \times 10^{-9} \mathrm{~J} / \mathrm{T}$ and $9 \mu \mathrm{m}$, which would have been nominally expected values given the particle radius $(9 \mu \mathrm{m})$ as determined from SEM images. This may be due to the limitations of the simple single dipole model that we use for $\mu P$. The injection spot is assumed to be a Gaussian of $5 \mu \mathrm{m}$ half-width, which is close to the the values obtained from fitting the camera image data to a $2 \mathrm{D}$ Gaussian. 


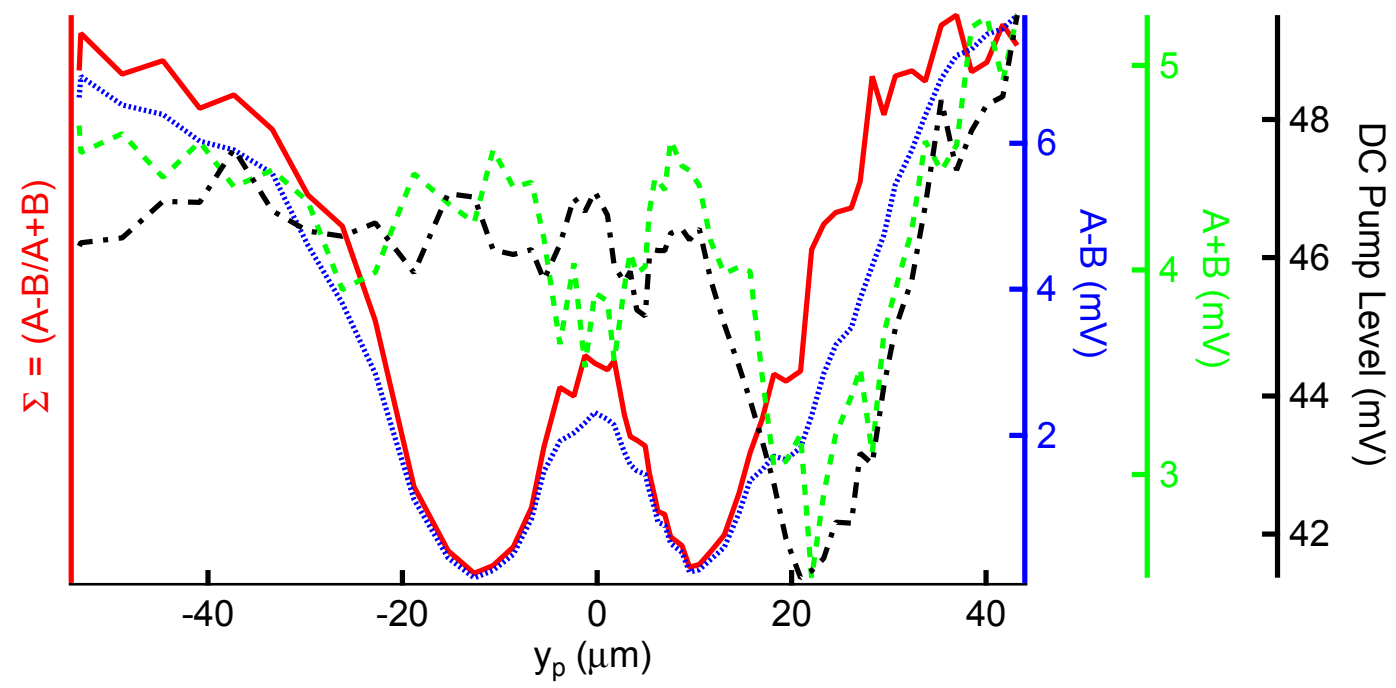

FIG. S4. Background removal and normalization of spin signal $\Sigma$. All the data shown in the figure correspond to the line scan data shown in Fig. 3 of the main text $\left(B_{t}=0\right.$, scan along $\hat{\mathbf{x}}$ ). Two lock-in signals $A-B$ (blue dashed line, right axis) and $\mathrm{A}+\mathrm{B}$ (green dashed line, right axis) are measured, where $\mathrm{A}$ and $\mathrm{B}$ correspond to the two photodiodes in the diode-bridge circuit. These correspond to the difference and sum of the intensities of the two circularly polarized components of the PL. The spin density is then proportional to $\mathrm{A}-\mathrm{B} / \mathrm{A}+\mathrm{B}$ (red solid line, left axis). The $\mathrm{A}-\mathrm{B}$ and $\mathrm{A}+\mathrm{B}$ channels can exhibit reflectivity changes (for e.g., the dip at $y_{P} 20 \mu \mathrm{m}$ ) as a function of the probe's position or due to the surface conditions of the sample. However, these are reasonably removed during the division process. Further evidence for the spurious reflectivity changes is seen in the spin-insensitive PL signal (black dashed line, right axis) collected by a separate photodiode.

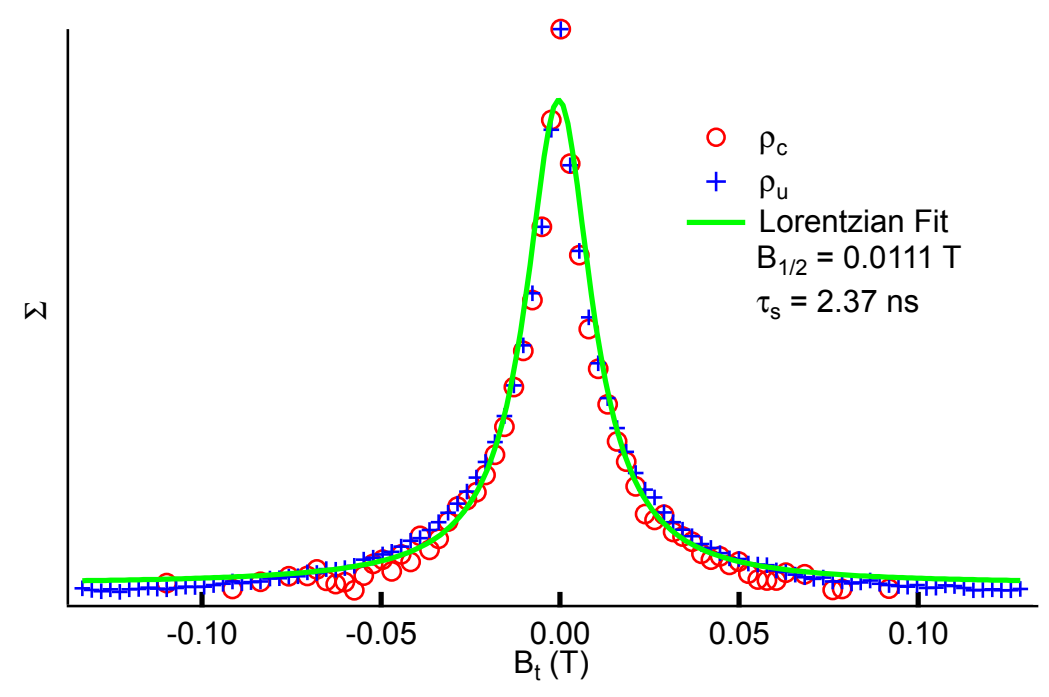

FIG. S5. Hanle curves measured far away from the $\mu P$ for the two spin density profiles $\rho_{c}$ (red circles) and $\rho_{u}$ (blue pluses).

\section{EXTRACTING THE SPIN DENSITY}

The PRFs presented in Fig. 4a and $\mathbf{b}$ were obtained by a Wiener deconvolution process,

$$
H_{B}\left(R, B_{t}\right)=\Sigma_{c}\left(\mathbf{r}_{p}, B_{t}\right) \circledast \rho\left(\mathbf{r}_{c}\right) .
$$

The deconvolution was implemented using Mathematica with a regularization parameter of 20 .

The PRFs presented in Fig. 4c and $\mathbf{d}$ were calculated using Eqns. S7, S8 and S5, and the point dipole parameters used in sec.VI. Fig. S6 presents the $\rho_{u}$ obtained by deconvolving using the theoretical PRFs in the main text from 


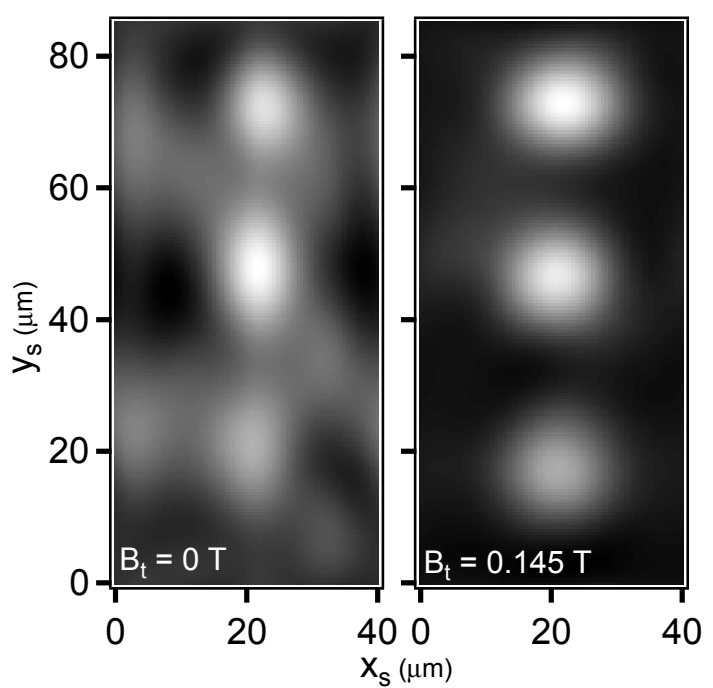

FIG. S6. spin density $\rho_{u}$ obtained from deconvolution of $\Sigma_{u}$ and the theoretical PRFs shown in Fig. 3a (left hand panel) and Fig. $3 \mathbf{b}$ (right hand panel) of the main text.

$\Sigma_{u}$

$$
\rho\left(\mathbf{r}_{c}\right)=\Sigma_{c}\left(\mathbf{r}_{p}, B_{t}\right) \circledast H_{B}\left(R, B_{t}\right) .
$$

These deconvolutions used a regularization parameter of 10 . 
* bhallamudi.1@osu.edu

$\dagger$ hammel@physics.osu.edu

1 M. Otsubo, T. Oda, H. Kumabe, and H. Miki, Journal of The Electrochemical Society 123, 676 (1976), http://jes.ecsdl.org/content/123/5/676.full.pdf+html, URL http://jes.ecsdl.org/content/123/5/676.abstract.

2 M. Furis, D. L. Smith, S. Kos, E. S. Garlid, K. S. M. Reddy, C. J. Palmstrom, P. A. Crowell, and S. A. Crooker, New J. Phys. 9, 347 (2007), URL http://iopscience.iop.org/1367-2630/9/9/347/. 